\title{
Microstructure evolution of hydrated cement pastes
}

\author{
A. Plassais, ${ }^{1}$ M.-P. Pomiès, ${ }^{1}$ N. Lequeux, ${ }^{1}$ J.-P. Korb,${ }^{2, *}$ D. Petit, ${ }^{2}$ F. Barberon, ${ }^{2}$ and B. Bresson ${ }^{3}$ \\ ${ }^{1}$ Laboratoire de Physico-Chimie des Polymères et Milieux Dispersés, UMR 7612 du CNRS, École Supérieure de Physique \\ et de Chimie Industrielles, 10, rue Vauquelin, 75231 Paris Cedex 05, France \\ ${ }^{2}$ Laboratoire de Physique de la Matière Condensée, UMR 7643 du CNRS, École Polytechnique, 91128 Palaiseau, France \\ ${ }^{3}$ Laboratoire de Physique Quantique, École Supérieure de Physique et de Chimie Industrielles, 10, rue Vauquelin, 75231 Paris Cedex 05, \\ France
}

\begin{abstract}
We propose an original method based on both proton nuclear magnetic relaxation dispersion and highresolution NMR spectra to investigate the microstructure of synthesized $\mathrm{Ca}_{3} \mathrm{SiO}_{5}$-hydrated cement paste. This method allows a clear assessment of the local proton chemical sites as well as the determination of dynamical information of moving proton species in pores. We show also how the microstructure evolves during and after completion of hydration in a range of length scales between 2 and $500 \mathrm{~nm}$. In particular, we show how the pore size distribution of the cement paste reaches progressively a power-law characteristic of a surface-fractal distribution with a dimension $D_{f}=2.6$, which takes into account the hierarchical order in the material. Last, we study how this pore size distribution is modified during setting by varying either the water-to-cement ratio or addition of ultrafine particles. This shows that our method could be relevant to relate the mechanical properties to the microstructure of the material. This proposed NMR method is general enough for the characterization of microstructure of any porous media with reactive surface involving water confinement.
\end{abstract}

\section{INTRODUCTION}

Porosity and microstructure are two very important structural characteristics of hydrated cement pastes [1-4]. They both influence the mechanical properties of materials as well as the transport efficiency of water and aggressive agents. A better control of the appearance and evolution of the microstructure during the hydration and setting is still needed to improve these properties. The microstructure is mainly characterized by the specific surface area, pore size distribution, tortuosity, and pores connectivity. However, exploring the porosity in this micropore range is so far poorly described because most of existing methods are invasive and perturbating [3].

Proton NMR measurements of in situ species are well suited to probe continuously the evolution of the material without perturbing the system. Here it is not needed to use nonwetting fluid intrusion or drying process that produce nanostructure modifications. Previous high-resolution NMR studies of cement pastes exhibit the different proton chemical species present in the phases of cement [5]. On the other hand, all the nuclear relaxation works on cement pastes observed several distinct relaxation rates that were attributed to the existence of different pore sizes [6-12]. However, it might be argued that these distinct relaxation rates correspond to different proton chemical species rather than to different pore sizes. This apparent interpretive contradiction is of significant importance to be addressed on a more complete experimental basis. We solve the problem by combining two methods. The first method probes the frequency dependence

\footnotetext{
*Author for correspondence. Electronic address: jeanpierre.korb@polytechnique.fr
}

(or dispersion curves) of the proton relaxation in a large frequency range. This allows a clear discrimination of the slow motion of diffusive protons at the pore interface from either the fast molecular motions of water in bulk or dynamics at local chemical sites. The second method associates a highresolution NMR proton (HRNMR) spectroscopy and relaxation study at a given high frequency. Based on the different time scales of high-resolution spectroscopy and nuclear spin relaxation, we prove that, apart from some protons bounded to silanol species and Portlandite, all the other identified surface proton chemical species are in fast exchange with free proton water in pores. So the measured relaxation rates of each proton species contain the spatial information on pore size distribution.

We apply these two methods to answer still open questions concerning the evolution of the microstructure of cement pastes during setting and to follow continuously the influence of cement formulation on this microstructure. We use, as a representative model material, synthesized tricalcium silicate $\mathrm{Ca}_{3} \mathrm{SiO}_{5}$, which represents $60 \%-70 \%$ of industrial Portland cement. The hydration of $\mathrm{Ca}_{3} \mathrm{SiO}_{5}$ leads to the formation of a gel of calcium silicate hydrate nanocrystals, $\mathrm{CaO}_{x} \mathrm{SiO}_{2} n \mathrm{H}_{2} \mathrm{O} \quad(x=1.7, n=4)$ labeled $\mathrm{C}-\mathrm{S}-\mathrm{H}$, mixed with large crystals of calcium hydroxyde, $\mathrm{Ca}(\mathrm{OH})_{2}$, named Portlandite. The $\mathrm{C}-\mathrm{S}-\mathrm{H}$ gel, the main source of porosity of the hydrated cement paste with a total porosity of more than $20 \%$, is formed by a disordered assemblage of lamellar nanocrystallized particles (typical size of 5-50 nm [13]) with an internal porosity (about a water layer) and an interparticle porosity varying from $1 \mathrm{~nm}$ up to $10 \mu \mathrm{m}$. Basically, during progressive setting of the materials, a part of the mixing water reacts to form solid $\mathrm{C}-\mathrm{S}-\mathrm{H}$ hydrates and Portlandite. At the end of the setting, the material is a network of hydrates saturated with residual confined water [2]. 
We show that the microstructure evolves at early age (during setting) to a discrete pore size distribution in a range of length scales between 2 and $500 \mathrm{~nm}$. We provide evidence that the distribution for an aged cement paste (after completion of hydration) evolves to a power-law distribution characteristic of a fractal surface. Last, we present a detailed study of the influence of the main formulation parameters to the microstructure (water-to-cement ratio and ultrafine particle addition).

Our method is sufficiently general to follow the progressive setting of a reactive interface of any porous media due to its ability to discriminate the diffusive effects leading to topological information (surface-to-volume ratio) from the multi-chemical-site NMR responses on the surface.

\section{EXPERIMENTS}

\section{A. Materials}

The starting anhydrous material is $\mathrm{Ca}_{3} \mathrm{SiO}_{5}$ (labeled $\mathrm{C}_{3} \mathrm{~S}$ ) synthesized by calcination of stoechiometric mixture of $\mathrm{CaCO}_{3}$ and silica at high temperature $\left(1100{ }^{\circ} \mathrm{C}\right)$ with a variable amount of $\mathrm{Fe}_{2} \mathrm{O}_{3}$. $\mathrm{Ca}_{3} \mathrm{SiO}_{5}$ was ground to a mean particle size of $10 \mu \mathrm{m}$. Two anhydrous starting materials were prepared with iron oxide $\left(\mathrm{Fe}_{2} \mathrm{O}_{3}\right)$ in a mass ratio of $0.05 \%$ (labeled in the text as $\mathrm{C}_{3} \mathrm{~S}-a$ ) and $0.7 \%$ (labeled as $\mathrm{C}_{3} \mathrm{~S}-b$ ). The latter corresponds to the iron content found in substitution in $\mathrm{Ca}_{3} \mathrm{SiO}_{5}$ of industrial Portland cement.

The $\mathrm{Ca}_{3} \mathrm{SiO}_{5}$ paste is obtained by mixing the anhydrous powder with distilled water with a water-to-cement mass ratio varying from $w / c=0.3$ to 0.7 in a sealed mold at room temperature. After $24 \mathrm{~h}$, the material is kept in saturated lime water solution during a delay ranging from hours to months. This process prevents the self-desiccation of the pore network. To specifically follow the early age of hydration by in situ NMR, cement paste was directly cured in the NMR rotor. In some samples, a small amount of fumed silica (6\% mass ratio) of average particle mean size of $0.15 \mu \mathrm{m}$ was added prior to hydration. The fumed silica is a common additive in cement materials because it reduces the amount of fragile crystals of Portlandite by reaction to form new $\mathrm{C}-\mathrm{S}-\mathrm{H}[2]$.

\section{B. Methods}

The first method gives the nuclear magnetic relaxation dispersion (NMRD) data, from nonresolved static spectra, on a very large range of Larmor frequencies. For that purpose, we used three different NMR techniques.

(i) The first technique is based on the well-known inversion-recovery NMR sequence used for $T_{1}$ measurements [14], which is usually written as the successive $\pi-\tau-\pi / 2$ pulses. These $T_{1}$ experiments are carried out on three Bruker spectrometers ASX 100, 300, and $500 \mathrm{MHz}$, referring to the Larmor frequency of the proton in 2.5, 7, and $12 \mathrm{~T}$ constant magnetic fields, respectively. The dispersion curves are completed by inversion-recovery measurement at a field ranging from 0.1 up to $1.5 \mathrm{~T}$ in a low-field homemade spectrometer. With these techniques, the high-field part of the dispersion curve is obtained. The three high-field spectrom- eters described above are equipped with a probe designed for solid-state NMR.

(ii) To explore the very-low-frequency part of the $T_{1}$ NMRD with the sensitivity of a high field, we measured on a $500-\mathrm{MHz}$ spectrometer the longitudinal relaxation time in the rotating frame $\left(T_{1 \rho}\right)$ in the frequency range of $10-100 \mathrm{kHz}$ [14].

(iii) The third technique requires a fast-field cycling spectrometer (Stelar) where the polarization and acquisition magnetic fields are 0.5 and $0.25 \mathrm{~T}$, respectively while the evolution magnetic field ranges from $0.25 \mathrm{mT}$ to $0.5 \mathrm{~T}$.

The second method (HRNMR) uses the high-resolution solid-state NMR magic angle spinning (MAS) at $500 \mathrm{MHz}$. This technique is used to suppress the effect of dipole-dipole interactions that prevent obtaining high-resolution spectra. The sample was spun at $30 \mathrm{kHz}$ in a $2.5-\mathrm{mm}$ rotor, and the spectra were acquired with a $4-\mu$ s pulse duration. We correlate this high-resolution spectroscopy with an inversion recovery sequence for measuring the spin-lattice relaxation time $T_{1}$ for the various proton species.

The degree of hydration (percentage of $\mathrm{C}-\mathrm{S}-\mathrm{H}$ formed) was obtained from quantitative $\mathrm{X}$-ray powder diffraction by measuring the amount of anhydrous $\mathrm{Ca}_{3} \mathrm{SiO}_{5}$ remaining along the curing time.

The amount of paramagnetic ferric ions was evaluated through an electron spin resonance (ESR) Bruker spectrometer operating at $9.6 \mathrm{GHz}$. The calibration of the ESR spectrum has been made by adding to our sample a small single monocrystal of $\mathrm{CuSO}_{4}$ with a precisely measured mass. The ESR $\mathrm{Fe}^{3+}$ peak is well resolved at room temperature for the synthesized $\mathrm{C}_{3} \mathrm{~S}-b$ that allows us to measure the concentration of $\mathrm{Fe}^{3+}$ ions. The synthesized $\mathrm{C}_{3} \mathrm{~S}-a$ was made with an order of magnitude less of iron oxide. Due to the bad resolution of the ESR spectrum, we assume that the amount of $\mathrm{Fe}^{3+}$ varies in the same ratio of the amount of iron oxide added.

\section{Magnetic relaxation data treatment}

Figure 1(a) shows a typical nonexponential normalized magnetization recovery displayed on a semilogarithmic scale. This relaxation curve is interpreted as a sum of $n$ decreasing exponential decays, each of them being defined by two parameters: the weight factor of magnetization $A_{i}$ and the relaxation time $T_{1 i}$, respectively. To extract this couple of parameters from the data, we used two different methods: (i) the Laplace inversion method [15] using the CONTIN program [16] and (ii) a discrete method known as curve peeling, consisting in a decomposition by an iterative fit of the longest $T_{1}$ component. The different steps of the curve-peeling decomposition are displayed on the Figs. 1(a) and 1(b). In first, the long-time behavior of the raw curve (labeled 1) in the Fig. 1(a) is fitted by an exponential and a new curve is obtained by subtracting this exponential fit. The same process is then applied iteratively. At each iterative step, it is carefully checked that the long-time behavior of the new curve is really exponential like. The CONTIN method applied to the raw data yields also a discrete couple set $\left(A_{i}, T_{1 i}\right)$ as shown in Fig. 1(c). For both methods, careful attention has been given 

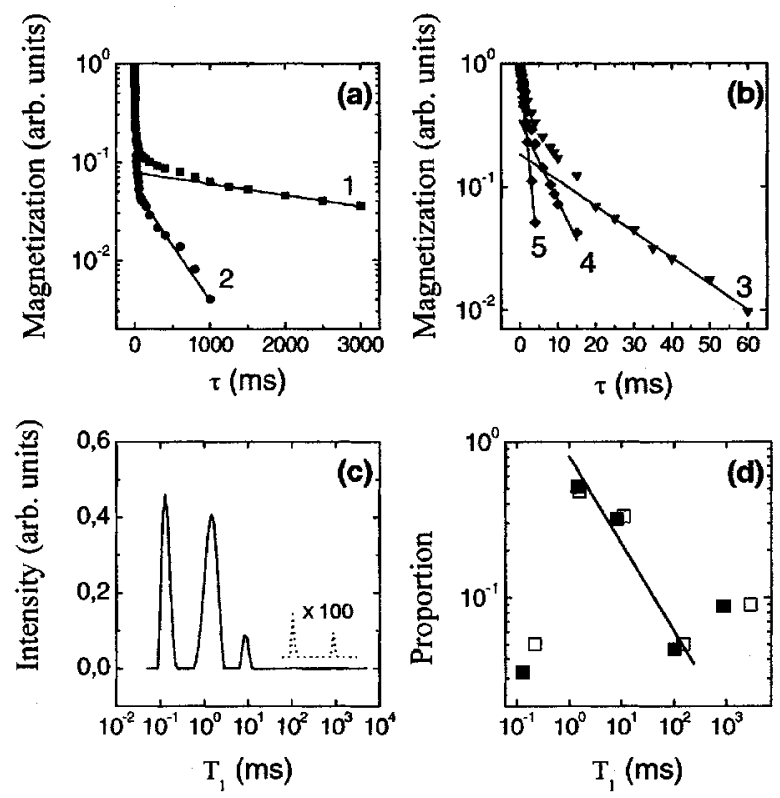

FIG. 1. (a) Different steps of the "curve-peeling" method used to extract successively five exponential components from the magnetization raw data of $\mathrm{a} \mathrm{C}_{3} \mathrm{~S}-b$ hydrated 1 month with $w / c=0.4$ at $20{ }^{\circ} \mathrm{C}$ (curve 1). The curves are displayed on a semilogarithmic scale. At the first step, a single exponential is subtracted to the raw data labeled 1; the result is the curve labeled 2. (b) The same method is iteratively applied up to the fifth step. (c) Semilogarithmic plot of continuous weight factor $A_{i}$ versus relaxation times $T_{1 i}$ obtained by CONTIN on the raw magnetization data 1 . (d) Comparison of the curve-peeling points ( $\square$ ) and the CONTIN points ( $\square$ ) representing the weight factors $A_{i}$ of the $i$ th exponential versus the relaxation time $T_{1 i}$ displayed on a double-logarithmic scale for a $\mathrm{C}_{3} \mathrm{~S}-b$ hydrated 1 month with $w / c=0.4$ at $20^{\circ} \mathrm{C}$. The values are extracted from the relaxation curve obtained by inversion recovery at $500 \mathrm{MHz}$. We note that both methods give the same result. The solid line exhibits a power law of exponent -0.58 for the discrete distribution of pore sizes over two orders of magnitude in $T_{1}$.

to the choice and density of the number of experimental points as well as to the signal-to-noise ratio of the raw curves. We check that the treatment is stable and both methods end up to the same couple set $\left(A_{i}, T_{1 i}\right)$ as seen in Fig. $1(d)$.

\section{RESULTS AND DISCUSSION}

\section{A. $T_{1}$ distribution of the hydrated cement paste}

The interpretation of the relaxation curves in Fig. 1(d) is based on the general biphasic fast-exchange model between the protons transiently belonging to the surface and the bulk in a given pore [17]. This model assumes that the molecular exchange between these two phases is faster than individual proton relaxation times. The overall proton relaxation rate is written as

$$
\frac{1}{T_{1}}=\frac{f_{\text {bulk }}}{T_{1}^{\text {bulk }}}+\frac{f_{\text {surf }}}{T_{1}^{\text {surf }}},
$$

where $T_{1 \text { bulk }}$ and $T_{1 \text { surf }}$ are the proton relaxation times in the bulk and at the surface, respectively. In Eq. (1), $f_{\text {surf }}=1-f_{\text {bulk }}$ and $f_{\text {bulk }}$ are the volume fractions of the surface and bulk phases, respectively. The bulk relaxation rate being much lower than the surface relaxation rate, Eq. (1) thus simplifies to

$$
\frac{1}{T_{1}} \approx \frac{\varepsilon S}{V} \frac{1}{T_{1}^{s u r f}},
$$

where $\varepsilon$ is a water thickness of about three water molecule layers [18] and with the surface-to-volume ratio $S / V$ that is inversely proportional to the average pore size $\langle R\rangle$. The discrete distribution of relaxation rates observed while analyzing the relaxation curve can thus be related to a discrete distribution of classes of pores $\left\langle R_{i}\right\rangle$ of weight factor $A_{i}$. In Fig. 1(d), the discrete distribution of pore sizes exhibits a power-law behavior (displayed as a solid line) over two orders of magnitude in $T_{1}$. This power law thus relates the amount of protons $A_{i}$ to the average pore size $\left\langle R_{i}\right\rangle$. One notes that the amount of protons decreases as a power law when the pore size increases. This behavior is a general trend for our aged samples and is also found in various cementitious materials $[10,12]$. From a mathematical point of view, a power law is also expected in the computation of the surface distribution $S(R)$ of a hierarchical distribution of noncommunicating or badly communicating spherical pores, $S(R) \propto\langle R\rangle^{2-D_{f}}$, of sizes ranging between $\langle R\rangle$ and the maximal pore radius $\left\langle R_{\max }\right\rangle(12)$. Here $D_{f}$ is the fractal dimension of the surface. As $2 \leqslant D_{f} \leqslant 3, S(R)$ diverges in the range of low radii. On the other hand, the expression of the volume distribution $V(R)$ as a function of $\langle R\rangle$ smoothly converges according to the relation $V(R) \propto\langle R\rangle^{3-D_{f}}$. The power-law [solid line in Fig. 1(d)] is thus characterized by an exponent $p$, which can be related to the surface fractal dimension $D_{f}\left(p=2-D_{f}\right)$. One finds $D_{f}=2.6$ in close agreement with small-angle x-ray and neutron scattering data [19]. The experimental points [Fig. 1(d)] with $T_{1}$ values of $10^{-1}$ and $10^{3} \mathrm{~ms}$, which are out of the power-law fit, correspond to silanol and Portlandite, respectively, and will be discussed below.

\section{B. Nuclear magnetic relaxation dispersion}

Figure 2 shows the NMRD curves $1 / T_{1}=f(\omega)$ obtained after curve peeling method of an one-year-aged $\mathrm{C}_{3} \mathrm{~S}-a$ sample. The renormalization of all these dispersion curves to a single one, shown in the inset of Fig. 2, proves that the same relaxation process occur at each of the four classes of $1 / T_{1 i}$ from 1 to $4000 \mathrm{~s}^{-1}$.

The benefit of exploring the range of low frequency is to isolate the typical NMRD dispersion features of the surface contribution of $1 / T_{1 i}$ associated with the different processes of molecular surface dynamics characterized by long correlation times, like molecular reencounters with the paramagnetic $\mathrm{Fe}^{3+}$ impurities. On the other hand, exploring the highfrequency range allows us to access to nuclear paramagnetic relaxation sensitive to very short electronic correlation time. The solid lines in Fig. 2 are the best fits obtained with a relaxation theory that considers two relaxation mechanisms occurring in very different frequency ranges. At low frequen- 


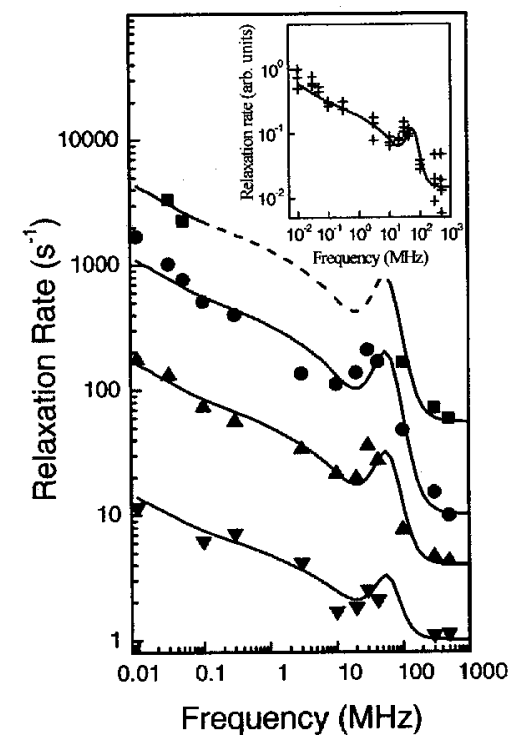

FIG. 2. Logarithmic plot of the dispersion curves showing the four longitudinal relaxation rates $1 / T_{1}$ versus the external magnetic field expressed in terms of the Larmor frequencies of the proton for a $\mathrm{C}_{3} \mathrm{~S}-a$ paste hydrated 1 year with $w / c=0.4$ at room temperature. The four curves have been successfully renormalized in the inset. The solid lines correspond to the best fit obtained by the relaxation model using the Ref. [4].

cies, the relaxation is due to the modulation of dipole-dipole interaction between the two-dimensional diffusing proton species and the $\mathrm{Fe}^{3+}$ ions fixed at the surface $[20,21]$. At high frequencies, the electron paramagnetic relaxation, controls the proton relaxation $[22,23]$. This mechanism leads to a characteristic peak observed in the $10-100 \mathrm{MHz}$. The application of this model to the cement-based materials has been described by Barberon et al. [4] where an analytical expression is proposed for the relaxation rate of protons $1 / T_{1 i}$. The frequency dependence of the overall spin-lattice relaxation rate, mainly due to the surface contribution, shows that the renormalization factors for the four classes of $1 / T_{1 i}$ are proportional to the surface-to-volume ratio. Providing that the amount of paramagnetic ferric ions is evaluated through a calibrated ESR experiment, these renormalization factors lead to the following values of average pore sizes $\left\langle R_{i}\right\rangle: 1.8$, $7.0,50$, and $600 \mathrm{~nm}$. Similar values were found for a oneyear-aged $\mathrm{C}_{3} \mathrm{~S}-b$ sample that contains an order of magnitude more paramagnetic ions than the one-year-aged $\mathrm{C}_{3} \mathrm{~S}-a$ shown in Fig. 2.

\section{High-resolution and relaxation results}

It might be argued that the different relaxation rates obtained above from NMRD data correspond to protons characteristic of different chemical species rather than to different pore sizes. Here we propose an original quantitative method based on both proton high-resolution spectra and spin-lattice relaxation data to properly assign the local proton chemical sites and the dynamical information of moving proton species in closed pores. In favor of this argument, it is known that different proton species $(\mathrm{CaOH}, \mathrm{SiOH}, \mathrm{HOH})$

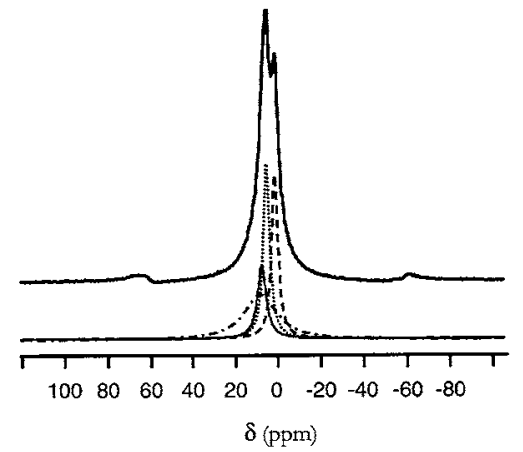

FIG. 3. NMR single-pulse proton MAS spectrum of $\mathrm{C}_{3} \mathrm{~S}-b$ hydrated 3 months with $w / c=0.4$ at $20{ }^{\circ} \mathrm{C}$ obtained at $12 \mathrm{~T}$ at a spinning frequency of $30 \mathrm{kHz}$. The decomposition shows four components: $\mathrm{Ca}-\mathrm{OH}$ bonds at $\delta=1 \mathrm{ppm}$, water at $\delta=5 \mathrm{ppm}$, and two different $\mathrm{Si}-\mathrm{OH}$ bonds at $\delta=7.6 \mathrm{ppm}$ and $\delta=8 \mathrm{ppm}$.

exist in cement paste. This is precisely what we find with high-resolution NMR while spinning the sample at the magic angle to get resolved spectra (Fig. 3). However, we show below that correlation between high-resolution NMR and nuclear relaxation on the same experiment (Fig. 4) rules out such an argument.

Another interest of such a correlation is to properly assign the high-resolution spectrum of Fig. 3. In Fig. 4, the inversion recovery proves that there are more than two peaks in the high-resolution spectrum of Fig. 3. Four peaks are requested to properly fit all the spectra displayed in the stack plot of Fig. 4. The following four proton species are usually assigned from their chemical shifts in accordance to Ref. [24].

(i) The large peak at $8 \mathrm{ppm}$, labeled No. 1, corresponds to protons characteristics of $\mathrm{SiOH}$ bindings.

(ii) The narrow peak at $7.6 \mathrm{ppm}$, No. 2, corresponds to protons characteristics of either $\mathrm{SiOH}$ or $\mathrm{HOH}$ in confined water bindings,

(iii) The narrow peak at $5 \mathrm{ppm}$, No. 3, corresponds to protons characteristics of $\mathrm{HOH}$ bindings, in majority capillary water (free water),

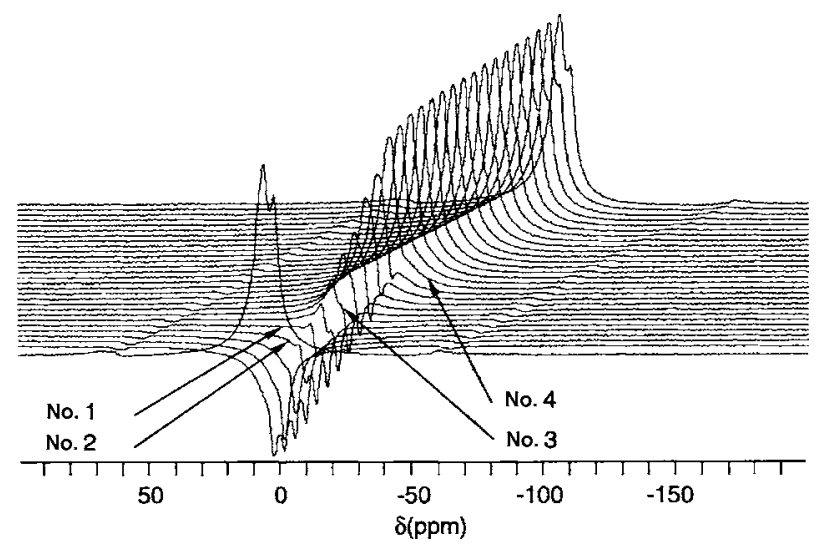

FIG. 4. Stack plots of inversion-recovery MAS-NMR spectra of $\mathrm{C}_{3} \mathrm{~S}-b$ hydrated 3 months with $w / c=0.4$ at $20^{\circ} \mathrm{C}$ obtained at $12 \mathrm{~T}$ and at a spinning frequency of $30 \mathrm{kHz}$. For each peak labeled Nos. 1-4 in the text, we locate with an arrow the locations where the signal comes to zero in this inversion-recovery experiment. 
(iv) The narrow peak at $1 \mathrm{ppm}$, No. 4, corresponds to protons characteristics of $\mathrm{CaOH}$ bindings, either in the Portlandite phase or in the $\mathrm{C}-\mathrm{S}-\mathrm{H}$.

The analysis of the inversion recovery data of Fig. 4 presents the following relaxation features.

(i) Peak No. 1 presents a monoexponential magnetization recovery (with $T_{1}=1 \mathrm{~ms}$ ).

(ii) Peaks No. 2 and No. 3 relax to equilibrium with a multiexponential distribution of $T_{1 i}$ similar to the one found from static NMRD data.

(iii) Peak No. 4 follows also a similar behavior as peaks Nos. 2 and 3, but presents a more pronounced longrelaxation-time component about $2 \mathrm{~s}$.

At this level, one has to consider the mobile and immobile proton species. The monoexponential behavior of the large peak No. 1 is characteristic of fluctuations of protons bounded to silanol species probably located within the intralayers of $\mathrm{C}-\mathrm{S}-\mathrm{H}$ [25]. The long-relaxation-time component of peak No. 4 corresponds to the proton involved in the $\mathrm{Ca}-\mathrm{OH}$ group of Portlandite whose relaxation is monoexponential (of several seconds). This result is consistent with what we obtained for a pure $\mathrm{Ca}-\mathrm{OH}$ crystalline phase. We observe that the peaks Nos. 2 and 3 and the remaining rapid relaxation component of No. 4 behave the same way: namely, a multiexponential behavior characteristic of mobile proton species in restricted pore spaces.

Now we address the question concerning the observation of a well-resolved NMR spectrum under the MAS condition while fulfilling the biphasic fast exchange model [Eq. (1)]. The time requirement

$$
t_{\text {spect }}=\frac{1}{2 \pi \Delta f} \approx 0.06 \mathrm{~ms} \leqslant t_{\text {res }}<T_{1, \text { fast }} \approx 1 \mathrm{~ms}
$$

specifies the limits in which a resolved proton NMR spectrum is compatible with the biphasic fast exchange model given in Eq. (1) between free proton water in pores and labyl surface proton species $(\mathrm{Ca}-\mathrm{OH}, \mathrm{Si}-\mathrm{OH}, \mathrm{H}-\mathrm{OH})$. Here $t_{\text {spect }}$ is the spectral time that is inversely proportional to the frequency difference between resolved lines $\Delta f, t_{\text {res }}$ is the time of residence of the labyl protons exchangeable among the various chemical sites $(\mathrm{Ca}-\mathrm{OH}, \mathrm{Si}-\mathrm{OH}, \mathrm{H}-\mathrm{OH})$ within the CSH, and $T_{1, \text { fast }}$ is the smallest of the spin-lattice relaxation times (of the order of the surface spin-lattice relaxation time).

On the other hand, the "long-lived" protons species ( $\mathrm{Si}-\mathrm{OH}$ or $\mathrm{Ca}-\mathrm{OH}$ ) belonging to the intralamellar structure of CSH are not moving and present a well-resolved and well-assigned NMR resonance. However, they are in intimate contact with a huge number of water molecules even in micropores. There results potentially another process of transfer between these two kinds of protons through a crossrelaxation process. This process involves spectral density $J^{(0)}\left(\omega_{I}-\omega_{I^{\prime}}\right)$ coming from the flip-flop dipolar contributions $\left(I^{+} I^{\prime-}+I^{-} I^{\prime+}\right)$ that transport the spin temperature of bulk proton to the "long-lived" protons species $\mathrm{Si}-\mathrm{OH}$ or $\mathrm{Ca}-\mathrm{OH}$ either on or at proximity to the pore surface. This could be relevant in the overall measured spin-lattice relaxation rate. Of course, a more quantitative calculation should be done,

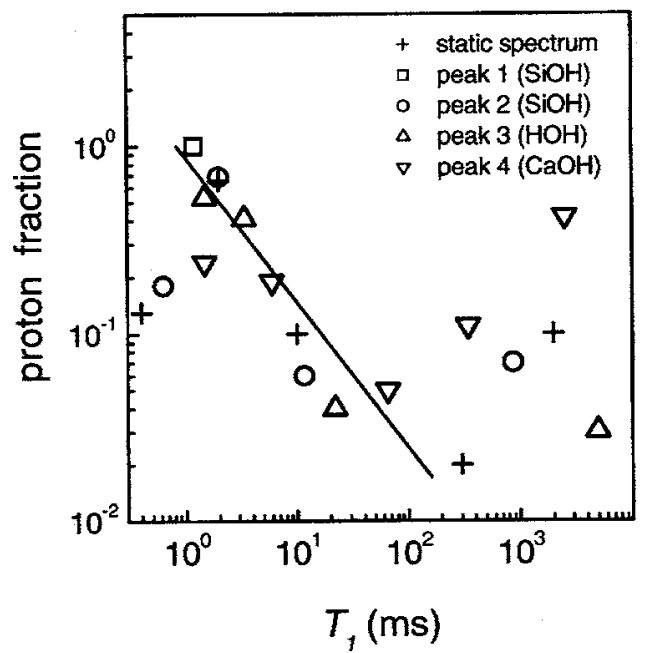

FIG. 5. Logarithmic plot of the proton fraction or pore size distribution, through the variation of their weight factor $A_{i}$ with the longitudinal relaxation time $T_{1}$ extracted from the $i$ th exponential recovery of each peak of data of Fig. 4. We note that, except for the large $\mathrm{Si}-\mathrm{OH}$ peak and $\mathrm{Ca}-\mathrm{OH}$ peak, all the other peaks present the same power-law distribution of exponent -0.66 , as in Fig. 1(d), characterized by the solid line. For each peak, one has $\sum_{i=1}^{N_{A}} A_{i}=1$, where $N_{A}$ is the number of $T_{1}$ for each proton species.

but an immediate consequence of this surface process is that it gives a less restrictive condition on the upper limit of Eq. (2) concerning the time of residence of "long-lived" protons.

One obtains from the raw magnetization recovery of each chemical species the function $A_{i}=f\left(T_{1 i}\right)$ corresponding to each species. In Fig. 5 are displayed the superposition of the distributions $A_{i}=f\left(T_{1 i}\right)$ associated with all the chemical species. The key result shown in this figure is that the distributions of the peaks 2, 3, and 4 as well as the static spectrum follow the same power-law dependence in a range of two orders of magnitude in $T_{1 i}$ between 1 and $200 \mathrm{~ms}$. This power law is similar to the one found from NMRD [Fig. 1(d)], which can be related to a surface fractal dimension $D_{f} \sim 2.6$. There are two physical significances of this result. (i) The nuclear relaxation for every proton species and in each class of pores is due to a pure restricted diffusion process of moving proton species in fast exchange with the proton bounded at the pore surface. (ii) The fractal dimension shows that the same relaxation process exists on different hierarchical length scales and that the species 2, 3, and 4 are present on all these length scales. On the contrary, if each relaxation rate was related to the local chemical site of a single species, one would observe four different monoexponential behaviors for the four peaks, which is not the case for species 2, 3, and 4. The specific relaxation mode of the peak at $1 \mathrm{ppm}$ shows a predominant relaxation mode for the long time $(2 \mathrm{~s})$. This can be simply explained by the fact that this peak is an exact superposition of two lines. The first line corresponds to the proton involved in the $\mathrm{Ca}-\mathrm{OH}$ group of Portlandite whose relaxation is monoexponential (of several seconds). The other line, whose relaxation is multiexponential, corresponds to the proton involved in the $\mathrm{Ca}-\mathrm{OH}$ groups of $\mathrm{C}-\mathrm{S}-\mathrm{H}$ which can interact with the other diffusing protons by exchange or cross relaxation. The long 


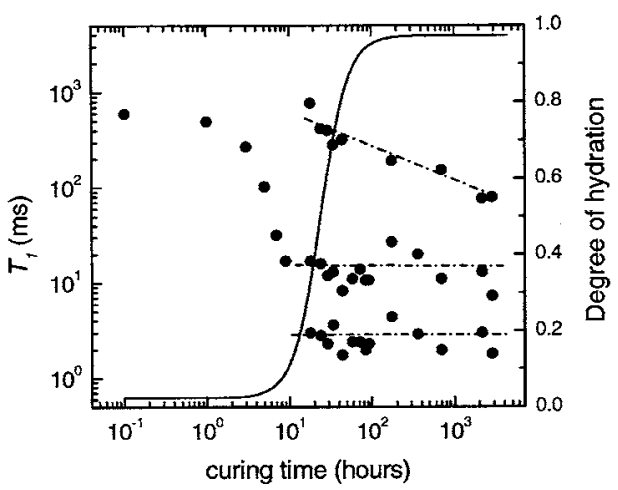

FIG. 6. Time evolution of the different $T_{1}$ values obtained at $500 \mathrm{MHz}$ (proportional to the average pore-size $\left\langle R_{i}\right\rangle$ ) for a $\mathrm{C}_{3} \mathrm{~S}-b$ sample hydrated with $w / c=0.4$ at $20{ }^{\circ} \mathrm{C}$. The time evolution of the hydration degree is also displayed on the figure by a solid line. Only the results associated with moving proton species in pores have been displayed.

spin-lattice relaxation time attributed to the Portlandite protons proves that protons belong to the solid-crystalline phase and rules out any exchange proton processes. The large peak No. 1 at $8 \mathrm{ppm}$ presents a monoexponential magnetization evolution (with $T_{1}=1 \mathrm{~ms}$ ) that can be simply explained by the bulk origin of this $\mathrm{SiOH}$ species within the $\mathrm{C}-\mathrm{S}-\mathrm{H}$.

In summary, this is the continuous spectroscopic control of the proton magnetization evolution to equilibrium of the different chemical species that has allowed us to discriminate the immobile species (hard-bounded $\mathrm{Si}-\mathrm{OH}$ and Portlandite) from the proton species relaxed by diffusive motions.

\section{Material results}

These methods have been used to follow the microstructure development during setting and hardening. The impact of water-to-cement ratio $(w / c)$ and of the addition of ultrafine particles onto the microstructure have been also investigated.

In first, these experiments allow to give an a posteriori comprehensive relationship between the NMRD relaxation data of Fig. 1(d) and the material characteristics. The shortest relaxation time is predominantly due to the large $\mathrm{Si}-\mathrm{OH}$ spectroscopic signal that corresponds to protons of the intralayers of the $\mathrm{C}-\mathrm{S}-\mathrm{H}$. The three following relaxation times are aligned along a power law that corresponds to three different classes of porosity, likely intercrystallite $\mathrm{C}-\mathrm{S}-\mathrm{H}$ pores. The pore size is given by our model considering that relaxation is induced by paramagnetic ions interacting with mobile proton species $(\mathrm{HOH}, \mathrm{SiOH}$, and $\mathrm{CaOH})$ in fast exchange with the bulk. The longest relaxation time is mainly due to the presence of the independent protons of Portlandite crystals.

This method has been used to follow the microstructure development during the setting of the hydrating paste on a large time scale. We present in Fig. 6, the variation of the $T_{1}$ distribution with the curing time. In the same figure is presented the variation of the degree of hydration with the curing time. In the induction period of hydration (before $1 \mathrm{~h}$ ),

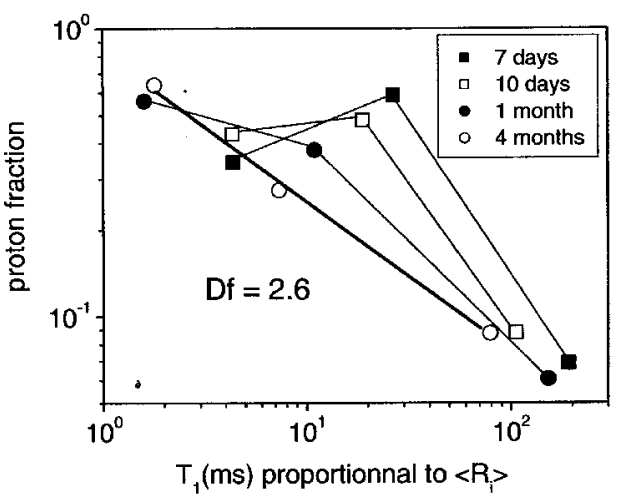

FIG. 7. Logarithmic plot of the pore size distribution for different periods of hydration from 7 days to 4 months. The distribution evolves progressively toward a power law $R^{2-D_{f}}$ after completion of hydration. This distribution is characteristic of a surface distribution where $D_{f} \approx 2.6$ is the surface fractal dimension that takes into account a hierarchical order in the texture of the material.

one has a full connected intergranular liquid network that induces a monoexponential relaxation curve with a characteristic time close to the bulk $T_{1}$ value of the interstitial solution. The net decrease of the $T_{1}$ between 1 and $10 \mathrm{~h}$ is due to the progressive increase of the $\mathrm{C}-\mathrm{S}-\mathrm{H}$ at the solid network. Such an increase of the solid-surface area induces a drastic enhancement of the number of proton species reencountered and consequently of the dipole-dipole spin-lattice relaxation rates. As seen in Fig. 6, this pure geometrical effects occurs before the acceleration of the degree of advancement of chemical reaction. This measure thus exhibits clearly the importance of the surface creation to enhance the occurrence of chemical reactions. After $10 \mathrm{~h}$, when the hydration speeds up, the magnetization decay is suddenly split into five components, three of them corresponding to the closing of the liquid network, which becomes disconnected after this threshold, and two of them corresponding to the bulk protons involved in Portlandite and $\mathrm{C}-\mathrm{S}-\mathrm{H}$. Therefore three classes of pores are present whose individual proportions are displayed in Fig. 7. The one corresponding to the longest $T_{1}$ value (macropores) progressively shrinks as the others conserve their mean pore radii between 10 and $2000 \mathrm{~h}$.

The leveling off observed for the degree of hydration after $100 \mathrm{~h}$ proves that the system is fully hydrated. Surprisingly, the evolution of the material continues after completion of hydration as seen in Fig. 7 where the distribution $A_{i}=f\left(T_{1 i}\right)$, for $\mathrm{C}_{3} \mathrm{~S}-b$ hydrated at room temperature, has been displayed for hydrating times ranging from 7 days to 4 months. Only the three exponentials corresponding to the three pore classes are considered in this time evolution. At the beginning of the hydration, the proton fraction in the intermediate class of pore dominates the total distribution. After 4 months, all the experimental points align on a power law characterized by an exponent $p$, which can be related to the surface fractal dimension $D_{f}\left(p=2-D_{f}\right)$. One finds $D_{f}$ ranging from 2.5 to 2.6 in close agreement with small-angle x-ray and neutron scatterings data [19]. The time evolution of the pore size distribution from a metastable state towards an equilibrium state characterized by a power law is representative of a hierarchi- 


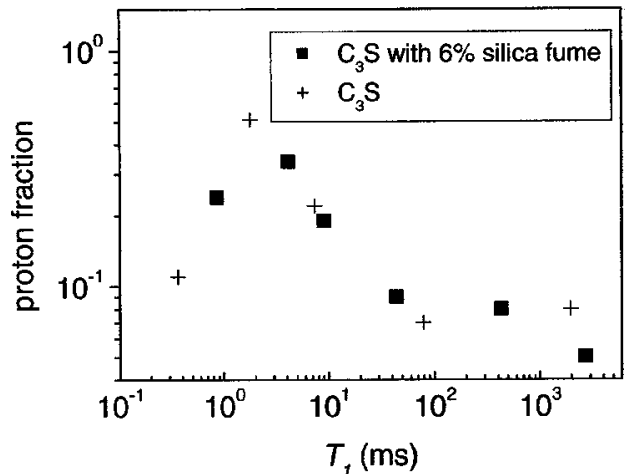

FIG. 8. Comparison of the pore size distribution for $\mathrm{C}_{3} \mathrm{~S}-b$ hydrated 4 months with and without fumed silica. When hydrated with fumed silica the water-to-solid ratio is 0.3 , a new exponential component appears, and the proportion of the pore with the largest $T_{1}$ decreases.

cal order that stabilizes the same texture on different length scales. This long-time texture evolution may be due to some rearrangements of $\mathrm{C}-\mathrm{S}-\mathrm{H}$ particles. Indeed, it is well known that the $\mathrm{C}-\mathrm{S}-\mathrm{H}$ particles are metastable, which might expose them to a redissolution-reprecipitation process [2].

We have also used this method to study the influence of additives as the fumed silica (SF) mixed with $\mathrm{Ca}_{3} \mathrm{SiO}_{5}$ at the beginning of hydration. Fumed silica is a well-known additive that induces a modification of the pore structure. At the beginning of hydration, SF locates between the cement particles and yields an improvement of the filling factor of the material [2]. During hydration, SF reacts with the Portlandite crystals to form porous $\mathrm{C}-\mathrm{S}-\mathrm{H}$. We investigate the effect of $\mathrm{SF}$ on the proton surface distribution of a $\mathrm{C}_{3} \mathrm{~S}-b$ paste hydrated during 4 months. The result is presented in Fig. 8. Two effects of SF are observed: (i) the decrease of the weight contribution of longest $T_{1}$ time (which is mainly characteristic of Portlandite) is due to the reaction of fumed silica with Portlandite, also called "pouzzolanic reaction," and (ii) the apparition of a new $T_{1}$ that appears when hydrated with fumed silica at $400 \mathrm{~ms}$ is due to a new class of pores.

The last formulation parameter which has been studied is the water-to-cement ratio $(w / c)$. We compare in Fig. 9 the proton surface distribution of a $\mathrm{C}_{3} \mathrm{~S}-b$ paste hydrated during 3 months with a $w / c$ of 0.3 and 0.7 . The surface proton distribution of the $\mathrm{Ca}_{3} \mathrm{SiO}_{5}$ paste hydrated with a ratio of 0.7 does not present the typical law power described previously. This could be related to the excess of free water compared to cement paste prepared with $w / c=0.3$ which modifies the pore size distribution with a displacement of the mean pore size toward higher values. This could be correlated with the very bad mechanical properties observed in this case. This shows that our method is relevant to correlate the mechanical properties to the texture of the material.

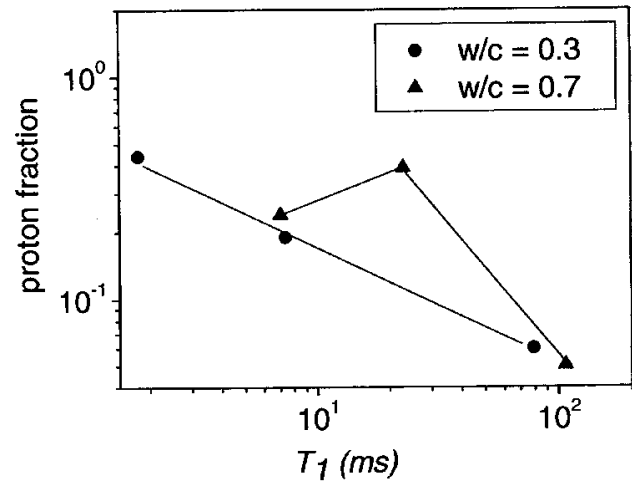

FIG. 9. Influence of the water-to-cement ratio $(w / c)$ on the distribution of pore size of a $\mathrm{Ca}_{3} \mathrm{SiO}_{5}$ paste hydrated during 3 months at $20^{\circ} \mathrm{C}$. There is no fractal distribution when $w / c=0.7$.

\section{CONCLUSION}

Based on nuclear magnetic relaxation dispersion and high-resolution NMR experiments on cement pastes, we propose an original non-perturbative way to properly separate the proton species population involved in the chemical bondings from those moving in closed pores. We deduce from these experiments a quantitative method to characterize the time evolution of the microstructure of cement paste on different length scales between 2 and $500 \mathrm{~nm}$. We use also this method to follow the progressive setting of the pore size distribution that evolves, after completion of hydration, to an equilibrium state characterized by a power law representative of a hierarchical order that stabilizes after 4 months the same texture on different length scales. This distribution is characteristic of a surface distribution where $D_{f} \approx 2.6$ is the surface fractal dimension that takes into account the hierarchical order in the texture of the material.

Last, the method is used to characterize the microstructure development during setting and hardening of cement paste and the impact of the water-to-cement ratio and the addition of fumed silica particles onto the modification of the microstructure. The results obtained show that our method is relevant to correlate the mechanical properties to the texture of the material. This method could be applied to any reactive porous materials with high surface area.

\section{ACKNOWLEDGMENTS}

This study has been supported both by CNRS and ATILH (Association Technique de l'Industrie des Liants Hydrauliques) through a research program on the description of the structure and properties of cement pastes (7, place de la Défense, 92974 Paris-La Défense Cedex). J.P.K. and D.P. thank S. Philippot for his pioneering work, which was at the origin of this study. 
[1] S. Diamond, Cem. Concr. Res. 29, 1181 (1999).

[2] H. F. W. Taylor, Cement Chemistry, 2nd ed. (Thomas Telford, London, 1997).

[3] J. J. Thomas, H. M. Jennings, and A. J. Allen, Concr. Sci. Eng. 1, 45 (1999).

[4] F. Barberon, J. P. Korb, D. Petit, V. Morin, and E. Bermejo, Phys. Rev. Lett. 90, 116103 (2003).

[5] R. J. Kirkpatrick and X. D. Cong, in Application of NMR Spectroscopy to Cement Science, edited by P. Colombet and A. R. Grimmer (Gordon and Breach, Amsterdam, 1994), p. 55.

[6] R. Blinc, G. Lahajnar, S. Zumer, and M. M. Pintar, Phys. Rev. B 38, 2873 (1988).

[7] W. P. Halperin, J. Y. Jehng, and Y. Q. Song, Magn. Reson. Imaging 12, 169 (1994).

[8] J. Y. Jehng, D. T. Sprague, and W. P. Halperin, Magn. Reson. Imaging 14, 785 (1996).

[9] A. J. Bohris, U. Goerke, P. J. McDonald, M. Mulheron, B. Newling, and B. Le Page, Magn. Reson. Imaging 16, 455 (1998).

[10] S. Philippot, J.-P. Korb, D. Petit, and H. Zanni, Magn. Reson. Imaging 16, 515 (1998).

[11] R. M. E. Valckenborg, L. Pel, K. Hazrati, K. Kopinga, and J. Marchand, Mater. Struct. 34, 599 (2001).

[12] C. Porteneuve, J. P. Korb, D. Petit, and H. Zanni, Cem. Concr. Res. 32, 97 (2002).
[13] S. Gauffinet, E. Finot, E. Lesniewska, and A. Nonat, C.R. Acad. Sci., Ser. IIc: Chim 327, 231 (1998).

[14] C. P. Slichter, Principles of Magnetic Resonance (SpringerVerlag, New York, 1989).

[15] P. Claverie, A. Denis, and E. Yeramian, Comput. Phys. Rep. 9, 249 (1989).

[16] S. W. Provencher, Comput. Phys. Commun. 27, 229 (1982).

[17] K. R. Brownstein and C. E. Tarr, Phys. Rev. A 19, 2446 (1979).

[18] J. J. Fripiat, M. Letellier, and P. Levitz, Philos. Trans. R. Soc. London, Ser. A 311, 287 (1984).

[19] A. J. Allen, J. J. Thomas, and H. M. Jennings, http:// www.aps.anl.gov/xfd/communicator/user2000/mat_sci.html

[20] J. P. Korb, M. W. Hodges, and R. G. Bryant, Phys. Rev. E 56, 1934 (1997).

[21] S. Godefroy, J. P. Korb, M. Fleury, and R. G. Bryant, Phys. Rev. E 64, 021605 (2001).

[22] I. Solomon, Phys. Rev. 99, 559 (1955).

[23] N. Bloembergen and L. O. Morgan, J. Chem. Phys. 34, 842 (1961).

[24] D. Heidemann, in Application of NMR Spectroscopy to Cement Science, edited by A. R. Grimmer (Gordon and Breach, London, 1994), p. 77.

[25] F. Brunet Ph. Bertand Th ${ }_{5}$ Charnentier, A. Nonat, and J. Virlet, 